\title{
STATISTICS OF ASSOCIATIONS AMONG IR GALAXIES
}

\author{
Jack F. Gallimore \\ and \\ William C. Keel \\ Dept. of Physics and Astronomy, University of Alabama
}

\section{Introduction}

In the course of expanding the search of Kleinmann et.al.(1988) for distant, infrared-luminous objects, we noticed (as is often remarked) that a large number of infrared-selected galaxies have close neighbors or show merger characteristics (e.g. tidal tails, distorted disks). Because the sample size is large (567 infrared galaxies and 2182 field galaxies), this sample is ideal for statistically examining the importance of interactions among infrared galaxies. In particular, we compare the nearest-neighbor distribution and the two-point correlation function of our sample with that of a control sample of field galaxies.

\section{II. - The Sample}

a) Selection Criteria

The IR galaxies in this sample are chosen from the IRAS Point-Source Catalog (PSC) (1985) such that they are detected at $60 \mu \mathrm{m}(F(60)>0.5 \mathrm{Jy})$, have nonstellar colors $(F(25) / F(60)<4)$, are southern sky objects with declination $-40^{\circ}<$ $\delta \leq-30^{\circ}$ (to make use of the ESO/SRC J films), and have galactic latitude $|b|>30^{\circ}$. Close-ups of these objects were made from ESO/SRC J sky survey films onto $35 \mathrm{~mm}$ film, with scale information preserved on each frame. If $\mathrm{J}$ films were unavailable (approx. 20\% of the sample), $\mathrm{R}$ films were used in place. The resulting individual fields covered on the average $24^{\prime} \times 18^{\prime}$ rectangles centered on each IR galaxy. In the event that more than one galaxy was found at the IRAS location, the optically brightest galaxy was (arbitrarily) chosen to be the IR galaxy. Because our analysis resolves angular separations no finer than 1', spuriously identifying an IR galaxy with a field galaxy at a separation of less than $1^{\prime}$ will have an insignificant or no effect on the final results. The field sample consists of all non-IR galaxies on each frame.

\section{b) The Data}

The angular dimensions of the bright core $\left(a_{1}, a_{2}\right)$ and the visible extent $\left(b_{1}, b_{2}\right.$, to the sky background) were measured for all sample galaxies. A weighted area, $A=\left(10\left(a_{1} a_{2}\right)+3\left(b_{1} b_{2}\right)\right) / 13$, was then calculated for each galaxy, and we computed a linear least-squares fit between $\log A$ and blue magnitudes $(B)$ obtained for 306 IR galaxies from Lauberts and Valentijn (1989). The resulting fit is good with an estimated standard deviation of 0.68 magnitude. Field galaxies fainter than 17 th 
magnitude are undersampled, but IR galaxies appear to be undersampled fainter than 15th magnitude. This is likely a result of the optical selection procedure; therefore, we maintain that the limiting magnitude of the entire sample is 17 .

\section{III. - Analysis}

The field and IR samples were divided into three magnitude-selected subsamples: $15 \leq B<16,16 \leq B<17$, and $15 \leq B<17$. Brighter galaxies were not considered because the maximum angular extent of the frames is smaller than the expected nearest-neighbor distance for $B<15$. To remove the effects of the IR sample, statistics for the field sample were only calculated from those frames where the IR galaxy did not belong to the corresponding sub-sample.

We calculated the angular correlation function $w(\theta)$ for each sub-sample by comparing the counts of galaxy pairs at a given angular separation to corresponding counts of pairs of points which were uniformly distributed over an identical geometry (Peebles, 1980, Peebles and Hauser, 1974). This technique has the advantage of removing edge effects due to the limited geometry. The standard deviation for each bin is estimated by $\sigma=(1+w(\theta)) / n_{p}$, where $n_{p}$ is the number of sample pairs with separation $\theta$. As shown by Sharp (1979), this is actually an over-estimate of $\sigma$. The estimates for $w$ are shown in figure 1 .

The nearest neighbor distribution $F_{n n}$ was calculated for each sub-sample using survival analysis techniques (Feigelson and Nelson, 1985). Whenever the distance from a galaxy to the field edge was smaller than the angular separation to that galaxy's nearest neighbor on a given frame, the distance to the field edge was taken as a lower limit to the true nearest-neighbor separation. With our data censored in this manner, we were able to estimate $F_{n n}$ using the Kaplan-Meier product-limit estimate and compare $F_{n n}$ for the field and IR samples using the logrank and Peto tests, which are relatively insensitive to the censoring distributions. To optimize the results for the field galaxies and eliminate double-counting of pairs, only the field galaxy closest to the center of a given field frame is used for these computations. The estimates for $F_{n n}$ are shown in figure 2, and the results of the logrank and Peto tests are given in table 1.

Table 1

\begin{tabular}{crcccccrrr}
\multicolumn{10}{c}{ Results of the Peto and Logrank Tests } \\
Group & $N_{I R}$ & $\%_{C}$ & $N_{F}$ & $\% C$ & Peto & $p$ & logrank & \multicolumn{1}{c}{$p$} \\
\hline $15 \leq B<16$ & 191 & 72 & 376 & 89 & 3.113 & 0.0019 & 3.174 & 0.0015 \\
$16 \leq B<17$ & 88 & 42 & 479 & 71 & 2.811 & 0.0049 & 3.110 & 0.0019 \\
$15 \leq B<17$ & 279 & 25 & 288 & 59 & 4.984 & $<0.0001$ & 5.473 & $<0.0001$
\end{tabular}

$N_{I R}$ and $N_{F}$ are the counts within each sub-sample of IR and field galaxies, respectively. $\%_{C}$ is the percentage of lower limits detected for for each preceding group. $p$ is the probability that both samples have the same parent distribution according to the preceding test. 

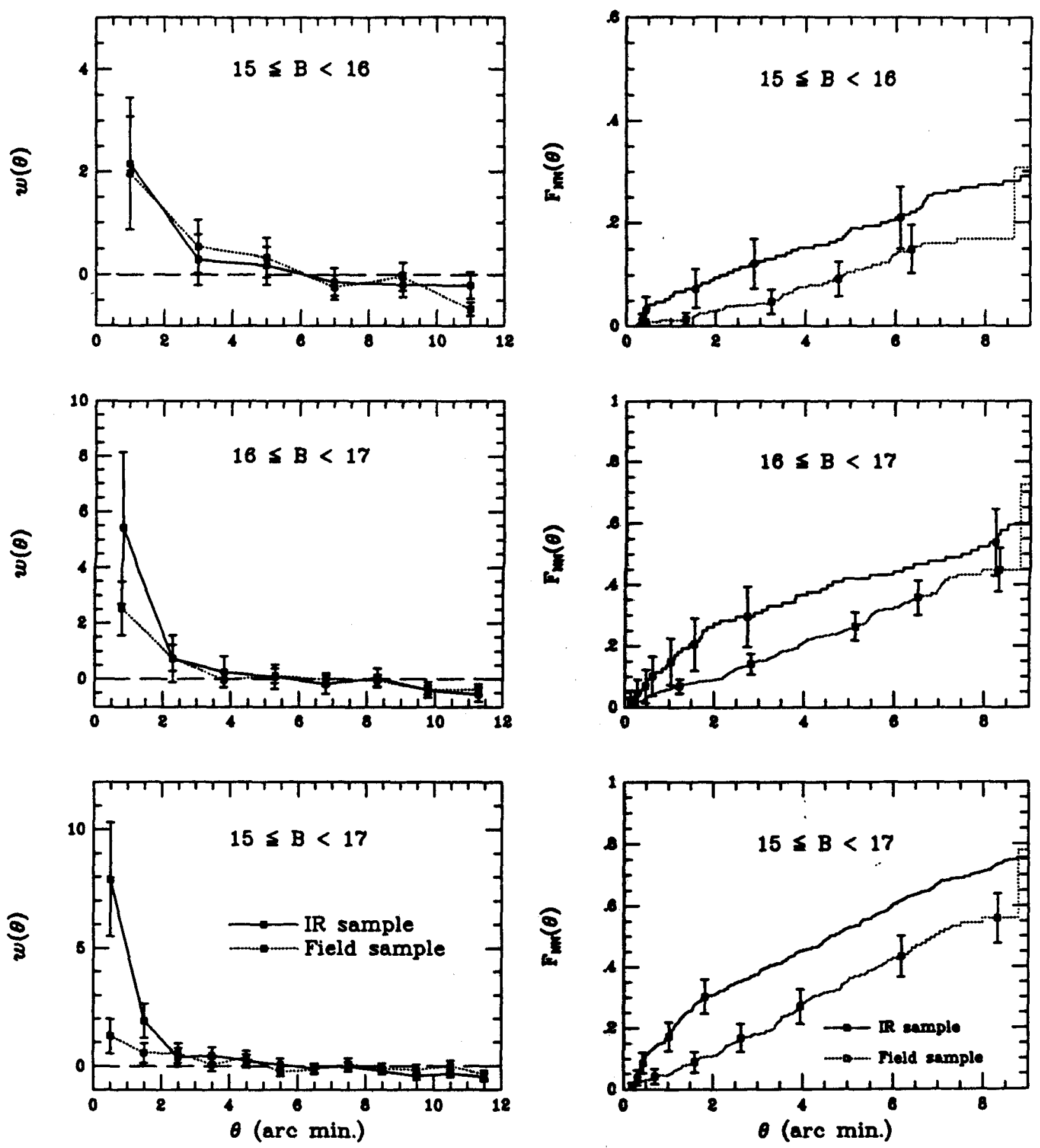

Figure 1

The estimated two-point angular correlation function for IR and field galaxies in each magnitude-selected group. Errorbars shown are $2 \sigma$.

Figure 2

The estimate of the cumulative nearest neighbor distribution function for IR and field galaxies in each magnitude-selected group. $2 \sigma$ errorbars are shown. 


\section{IV. - Results}

IR galaxies show the greatest enhancement in correlation for the $15 \leq B<17$ sample, where the restriction in pairing by magnitude is weaker, and the IR galaxies are better removed from the field statistics. Also, the IR galaxies in all three subsamples display an enhanced probability of pairing relative to the field sample as shown by the nearest-neighbor statistics.

A final consideration is whether the enhanced IR flux for these galaxies is actually related to physical interactions with neighboring galaxies or is simply the combined flux of several proximate galaxies of comparable brightness. In our survey, $4 \%$ of the IR galaxies with $15 \leq B<17$ had 3 or more neighbors within 2.5 arc minutes whose brightness differed with the IR galaxy by no more than 1 magnitude, so the percentage of galaxies spuriously identified as infrared bright is probably small. Therefore, we now have quantitative evidence that confirms the conventional (qualitative) wisdom: there is a strong tendency for galaxies with enhanced infrared luminosity to be found in physical associations.

\section{References}

Feigelson, E.D., and Nelson, P.I. 1985, Ap J., 293, 192.

IRAS Point Source Catalog 1985, Joint IRAS Science Working Group (Washington, D.C.: U.S. Government Printing Office).

Kleinmann, S.G., Hamilton, D., Keel, W.C., Wynn-Williams, C.G., Eales, S.A., Becklin, E.E., and Kuntz, K.D. 1988, Ap J.,328, 161.

Lauberts, A., and Valentijn, E.A. 1989, The Surface Photometry Catalogue of the ESO-Uppsala Galaxies (Garching bei München: European Southern Observatory)

Peebles, P.J.E. 1980, The Large Scale Structure of the Universe (Princeton: Princeton University Press.

Peebles, P.J.E., and Hauser, M.G. 1974, Ap J Suppl.,28, 19.

Sharp, N.A. 1979, Astr. Ap., 74, 308. 